\title{
Archivos \\ de Cardiología de México

\section{Estudio de la perfusión pulmonar posterior al cierre percutáneo del conducto arterioso permeable utilizando el dispositivo Amplatzer Duct Occluder en niños}

\author{
José Rafael Parra-Bravo a,*, Adriana Apolonio-Martínez ${ }^{\mathrm{b}}$, \\ María de Jesús Estrada-Loza ${ }^{a}$, Luisa Gracia Beirana-Palencia ${ }^{a}$ \\ y César Iván Ramírez-Portillo ${ }^{a}$
}

\begin{abstract}
a Servicio de Cardiología y Hemodinamia, Hospital de Pediatría Centro Médico Nacional Siglo XXI Instituto Mexicano del Seguro Social, México, Distrito Federal, México

b Servicio de Pediatría, Hospital de Pediatría Centro Médico Nacional Siglo XXI Instituto Mexicano del Seguro Social, México, Distrito Federal, México
\end{abstract}

Recibido el 14 de agosto de 2014; aceptado el 10 de diciembre de 2014

\author{
PALABRAS CLAVE \\ Conducto arterioso \\ permeable; \\ Cierre percutáneo; \\ Amplatzer Duct \\ Occluder; \\ Gammagrafía \\ pulmonar; \\ México
}

\begin{abstract}
Resumen
Objetivo: La reducción en la perfusión pulmonar se ha descrito tras el cierre percutáneo del conducto arterioso permeable con varios dispositivos. Evaluamos la perfusión pulmonar posterior al cierre percutáneo del conducto arterioso con el dispositivo Amplatzer Duct Occluder utilizando la gammagrafía pulmonar de perfusión.

Métodos: Treinta pacientes con oclusión transcatéter exitosa del conducto arterioso permeable utilizando el dispositivo Amplatzer Duct Occluder fueron incluidos en este estudio. La gammagrafía pulmonar de perfusión se realizó 6 meses después del procedimiento. Las velocidades de flujo máximo y la protrusión del dispositivo fueron analizadas por ecocardiografía Doppler. Una perfusión pulmonar izquierda $<40 \%$ se consideró anormal.

Resultados: El implante del dispositivo fue exitoso en todos. La media de perfusión hacia el pulmón izquierdo fue de $44.7 \pm 4.9 \%$ (37.8-61.4). Cinco pacientes (16.6\%) mostraron disminución en la perfusión pulmonar izquierda. Tanto la edad y el peso bajo, así como la longitud del conducto arterioso y la relación diámetro mínimo y máximo con la longitud del conducto arterioso fueron estadísticamente significativos en los pacientes con anomalías de la perfusión pulmonar. Se observó protrusión del dispositivo en 6 pacientes con una velocidad de flujo máximo mayor en la arteria pulmonar izquierda.
\end{abstract}

* Autor para correspondencia. T. Sánchez de la Barquera 108-304, Colonia Merced Gómez. Delegación Benito Juárez. CP 03930. México, DF., México. Teléfono: +5680 2281.

Correo electrónico: rafaelparrabravo@yahoo.com.mx (J.R. Parra-Bravo). 
Conclusión: La perfusión pulmonar izquierda puede comprometerse tras el cierre percutáneo del conducto arterioso con el Amplatzer Duct Occluder. El aumento en la velocidad de flujo en el origen de la arteria pulmonar izquierda puede ser un pobre indicador de la reducción en la perfusión pulmonar y puede ocurrir en ausencia de protrusión del dispositivo.

(C) 2014 Instituto Nacional de Cardiología Ignacio Chávez. Publicado por Masson Doyma México S.A. Todos los derechos reservados.

\section{KEYWORDS}

Patent ductus arteriosus; Percutaneous closure; Amplatzer Duct Occluder; Lung perfusion scan; México

\section{Introducción}

El cierre percutáneo del conducto arterioso permeable (CAP) es un procedimiento bien establecido y sus resultados inmediatos son bien conocidos ${ }^{1,2}$. Para el cierre de diferentes tipos y tamaños angiográficos del CAP se utiliza una amplia variedad de dispositivos ${ }^{3-5}$. A pesar de que los resultados del procedimiento intervencionista son generalmente satisfactorios, algunas complicaciones han sido informadas ${ }^{6}$. Distorsiones mecánicas de los vasos adyacentes, particularmente de la arteria pulmonar izquierda (API) ocurren con algunos dispositivos ${ }^{6-8}$. La protrusión del dispositivo hacía el origen de la API puede condicionar una reducción del flujo sanguíneo hacía el pulmón izquierdo ${ }^{9,10}$. Dessy et al. ${ }^{11}$ describen que el $14 \%$ de los pacientes sometidos a cierre percutáneo del CAP y evaluados por estudios de gammagrafía pulmonar muestran reducción en el flujo pulmonar izquierdo. El efecto de diferentes dispositivos sobre el flujo pulmonar, utilizando la gammagrafía pulmonar de perfusión ha sido descrito por otros autores ${ }^{12-15}$. Aunque el Amplatzer Duct Occluder I (ADO I; AGA Medical Corporation, EE. UU.) es el oclusor más utilizado en los CAP de un tamaño moderado a grande, su protrusión hacía la API y su impacto en la obstrucción y distribución del flujo sanguíneo no han sido completamente evaluados ${ }^{3-5,9,15}$.

Describimos el efecto del dispositivo Amplatzer Duct Occluder (ADO) en la obstrucción del origen de la API y la perfusión pulmonar izquierda, utilizando la gammagrafía pulmonar de perfusión.

\section{Métodos}

\section{Pacientes}

Se incluyeron 30 pacientes (20 niñas) en quienes se realizó cierre percutáneo del CAP utilizando el dispositivo ADO. Los pacientes seleccionados cumplían criterios clínicos y ecocardiográficos de un CAP de tamaño moderado y un peso mayor de $4 \mathrm{~kg}^{8,9}$. La edad al momento del cierre del CAP fue de $23.8 \pm 13.7$ meses (intervalo: $7-67$ meses) y el peso de $11.1 \pm 4.5 \mathrm{~kg}$ (intervalo: $4.1-25.5 \mathrm{~kg}$ ). Quince pacientes $(50 \%)$ evidenciaron un peso $\leq 10 \mathrm{~kg}$. Ocho pacientes (26.6\%) tenían otras malformaciones cardiacas asociadas: comunicación interventricular perimembranosa pequeña en 
Tabla 1 Datos demográficos y resultados del cateterismo en la población total, y comparación entre aquellos con perfusión normal y anormal

\begin{tabular}{llccc}
\hline Variable & Grupo total $(\mathrm{n}=30)$ & Perfusión normal $(\mathrm{n}=25)$ & Perfusión anormal $(\mathrm{n}=5)$ & Valor de $\mathrm{p}$ \\
\hline Edad (meses) & $23.8 \pm 13.7$ & $24.6 \pm 14.8$ & $19.6 \pm 5.6$ & 0.041 \\
Peso $(\mathrm{kg})$ & $11.1 \pm 4.5$ & $11.42 \pm 4.84$ & $9.3 \pm 1.8$ & 0.050 \\
Qp/Qs & $2.13 \pm 0.88$ & $2.14 \pm 0.90$ & $2.06 \pm 0.87$ & 0.356 \\
Características del CAP & & & & \\
$\quad$ Diámetro mínimo $(\mathrm{mm})$ & $2.7 \pm 1.4$ & $2.64 \pm 1.45$ & $2.76 \pm 1.1$ & 0.254 \\
Diámetro máximo $(\mathrm{mm})$ & $9.75 \pm 2.06$ & $9.74 \pm 2.26$ & $8.50 \pm 2.91$ & 0.207 \\
Longitud (mm) & $7.61 \pm 2.07$ & $8.16 \pm 1.77$ & $5.02 \pm 1.30$ & 0.023 \\
Relación diámetro mínimo/máximo & $0.26 \pm 0.1$ & $0.26 \pm 0.09$ & $0.27 \pm 0.1$ & 0.316 \\
Relación diámetro mínimo/longitud & $0.38 \pm 0.23$ & $0.35 \pm 0.21$ & $1.42 \pm 2.01$ & 0.019 \\
Relación diámetro máximo/longitud & $1.41 \pm 0.54$ & $1.27 \pm 0.42$ & $2.65 \pm 1.42$ & 0.001 \\
$\quad$ Tamaño del ADO (mm) & $5.07 \pm 1.46$ & $5.04 \pm 1.54$ & $5.20 \pm 1.09$ & 0.227 \\
Diferencia ADO-CAP (mm) & $2.33 \pm 0.54$ & $2.31 \pm 0.58$ & $2.42 \pm 0.19$ & $11 \pm 4.69$ \\
Gradiente máximo en API & $7.36 \pm 4.64$ & $6.40 \pm 4.17$ & 0.353 & 0.281 \\
\hline
\end{tabular}

ADO: Amplatzer Duct Occluder; API: arteria pulmonar izquierda; CAP: conducto arterioso permeable; Diferencia ADO-CAP: diferencia tamaño del ADO y diámetro mínimo del CAP; Qp/Qs: relación gasto pulmonar/sistémico.

2; foramen oval permeable en 3; coartación aórtica leve, estenosis de rama pulmonar izquierda y otra, uno en cada uno. Los datos demográficos y los resultados del cateterismo se muestran en la tabla 1. Previo al procedimiento intervencionista, se realizó un ecocardiograma Doppler-color para evaluar el gradiente de presión entre el tronco pulmonar y las arterias pulmonares, en la proyección del eje corto ${ }^{16}$.

\section{Protocolo de cierre del conducto arterioso permeable}

La técnica del cierre percutáneo del CAP ha sido descrita previamente por los autores ${ }^{8-10}$. El dispositivo se seleccionó en función del tamaño y morfología del CAP, de acuerdo con Krichenko et al. ${ }^{17}$ y de un diámetro de $2 \mathrm{~mm}$ mayor al diámetro mínimo del CAP.

\section{Seguimiento}

La evaluación ecocardiográfica transtóracica se realizó en todo paciente previo a su alta $(24 \mathrm{~h}), 3$ y 6 meses posteriores al procedimiento, enfatizando en la presencia de fuga residual, protrusión del dispositivo, y mediante evaluación Doppler se determinó la velocidad máxima $\left(V_{\text {máx }}\right)$ entre el tronco pulmonar y la API. Se consideró una $V_{\text {máx }}>1.6 \mathrm{~m} / \mathrm{s}$ (gradiente $>10 \mathrm{mmHg}$ ) como indicativa de posible estenosis en el origen de la API.

\section{Estudio de la perfusión pulmonar}

La gammagrafía pulmonar de perfusión se realizó 6 meses después del procedimiento utilizando una inyección intravenosa de macroagregados de albúmina marcada con ${ }^{99 \mathrm{~m}} \mathrm{Tc}$ a una dosis de $74 \mathrm{MBq} / 2 \mathrm{mCi}$ a través de una vena periférica en posición supina. El procedimiento se realizó en una cámara gamma (E-cam Siemens), con adquisición de imagen estática en proyección anterior y posterior durante $10 \mathrm{~min}$, para evaluar la distribución del flujo sanguíneo entre los pulmones. El porcentaje de perfusión en ambos pulmones se calculó al dividir la radiactividad media obtenida en ambas posiciones. Se consideró un flujo $<40 \%$ hacía el pulmón izquierdo como anormal $^{11-15}$. El protocolo de estudio fue aprobado por el comité de investigación local (R-2014-3603-7).

\section{Análisis estadístico}

Se realizó análisis estadístico descriptivo, con cálculos de medidas de tendencia central (media, mediana) y dispersión (desviación estándar, mínimo y máximo) para las variables cuantitativas de acuerdo con el tipo de distribución. Para las variables cualitativas, frecuencias y porcentajes. Los resultados se expresan como media \pm desviación estándar o mediana e intervalos. En el análisis de los datos se emplearon la prueba exacta de Fisher y la prueba de $\chi^{2}$ de Pearson. Para contrastar las variables cuantitativas, se utilizó la prueba de $U$ de Mann-Whitney. Todos los análisis se hicieron con el paquete estadístico SPSS versión 20 , considerando un valor de $\mathrm{p}<0.05$ con significación estadística.

\section{Resultados}

El implante del dispositivo fue exitoso en todos los pacientes (fig. 1) y la prevalencia de fuga residual posterior al implante del dispositivo fue del $30 \%$ y del $6.7 \%$ a las $24 \mathrm{~h}$. Ninguno de los pacientes mostró fuga residual 3 meses después del procedimiento. Por ecocardiografía Doppler, un paciente mostró una $V_{\text {máx }}>2 \mathrm{~m} / \mathrm{s}(23 \mathrm{mmHg})$ en la API antes del implante del dispositivo. Hemodinámicamente, observamos hipertensión arterial pulmonar $(P M P \geq 25 \mathrm{mmHg})$ en 12 pacientes (mediana $30 \mathrm{mmHg}$ ), siendo $>35 \mathrm{mmHg}$ en 3 de ellos. La relación gasto pulmonar/gasto sistémico mostró una mediana de 2.04/1Lt/min (intervalo: 1.2-4.7). Doce pacientes $(40.1 \%)$ evidenciaron un diámetro mínimo angiográfico del $C A P \leq 2 \mathrm{~mm}$ y 3 pacientes $(10 \%)$ mostraron una longitud del CAP $<5.8 \mathrm{~mm}$. El tipo de CAP encontrado fue: cónico (tipo A) en 21 casos (70\%), tubular (tipo C) en 5 casos (16.7\%) y tipo $E$ (cónico elongado) en 4 casos (13.3\%). 


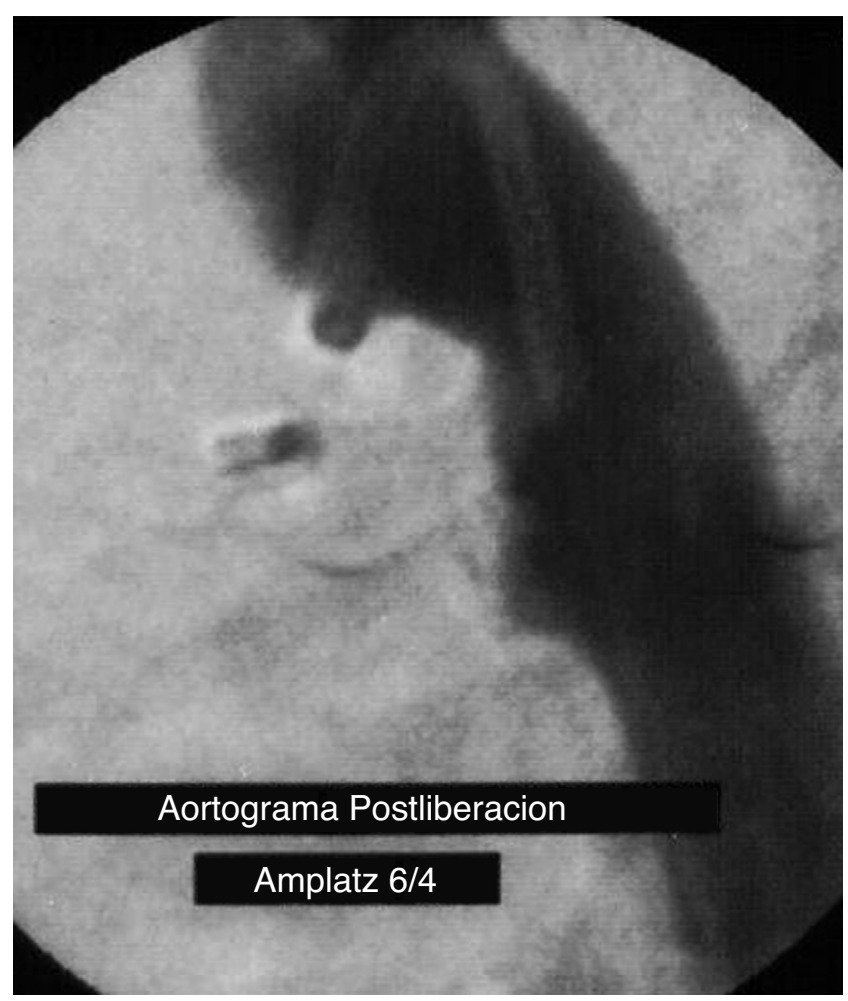

Figura 1 Aortografía postimplante del dispositivo Amplatzer Duct Occluder $6 / 4 \mathrm{~mm}$, posicionado completamente dentro del conducto arterioso y sin flujo residual.

En la sala de hemodinamia, un paciente mostró un gradiente de retiro de presión entre la API y el tronco pulmonar de $12 \mathrm{mmHg}$ previo al procedimiento y que persistió tras el implante del dispositivo. El tamaño del dispositivo fue $5 / 4$ en 12 casos (40\%), 6/4 en 5 casos (16.7\%), 8/6 en 11 casos (36.7\%), 10/8 en un paciente (3.3\%) y $12 / 10$ en otro (3.3\%). El tamaño del dispositivo más utilizado fue el $5 / 4$ y el $8 / 6(5.07 \pm 1.46 \mathrm{~mm})$ para una relación ADO/CAP para el grupo total de $2.1 \pm 0.7$. En 21 pacientes (70\%) se utilizó un dispositivo con un diámetro $>2 \mathrm{~mm}$ al diámetro mínimo del CAP con una media de $2.3 \pm 0.5$ en milímetros en la relación ADO/CAP.

Al analizar los resultados de la gammagrafía pulmonar, encontramos una mediana de porcentaje de perfusión para el pulmón derecho del 55.3\% (intervalo: 40.6-62.24) y para el pulmón izquierdo del 44.7\% (intervalo: 37.7-61.36). En 5 pacientes (16.6\%) se evidenció disminución en la perfusión del pulmón izquierdo, definida como $<40 \%$ del flujo pulmonar total (fig. 2). Se compararon las variables entre los pacientes con perfusión pulmonar normal y disminuida (tabla 1).

Los valores medios de edad y peso fueron significativamente menores en los pacientes con disminución en la perfusión pulmonar izquierda $(\mathrm{p}=0.04,0.05$, respectivamente). Aunque los diámetros mínimo y máximo del CAP fueron similares en los pacientes con y sin alteración en la perfusión pulmonar ( $p=0.25,0.20$, respectivamente), la longitud del CAP fue significativamente menor en los pacientes con disminución en la perfusión pulmonar izquierda $(p=0.04)$. La relación diámetro mínimo y máximo del CAP con su longitud, también fueron significativamente menores en aquellos pacientes con flujo pulmonar disminuido $(p=0.019$ y 0.001 , respectivamente).

La ecocardiografía Doppler postimplante del ADO evidenció 5 pacientes $(16.6 \%)$ con incremento en la $V_{\text {máx }}$ en la API (excluyendo al paciente con estenosis preimplante) con valores entre 1.7-2.2 $\mathrm{m} / \mathrm{s}$ (gradiente $11-19 \mathrm{mmHg}$ ) y con una disminución leve del flujo pulmonar (37-39\%) en 3 de ellos $(p=0.09)$. Seis pacientes $(20 \%)$ evidenciaron protrusión del dispositivo hacia la $\mathrm{API}$, con un gradiente $>10 \mathrm{mmHg}$ en 5 de ellos. Al comparar el resultado de la perfusión pulmonar entre los pacientes con y sin protrusión del dispositivo, no hubo diferencia estadísticamente significativa (tabla 2).

Se realizó correlación de Spearman entre el gradiente de la API y la perfusión pulmonar izquierda (fig. 3), observando que la menor perfusión pulmonar se relacionó con un mayor gradiente en la API (rho -0.530), sin embargo, no

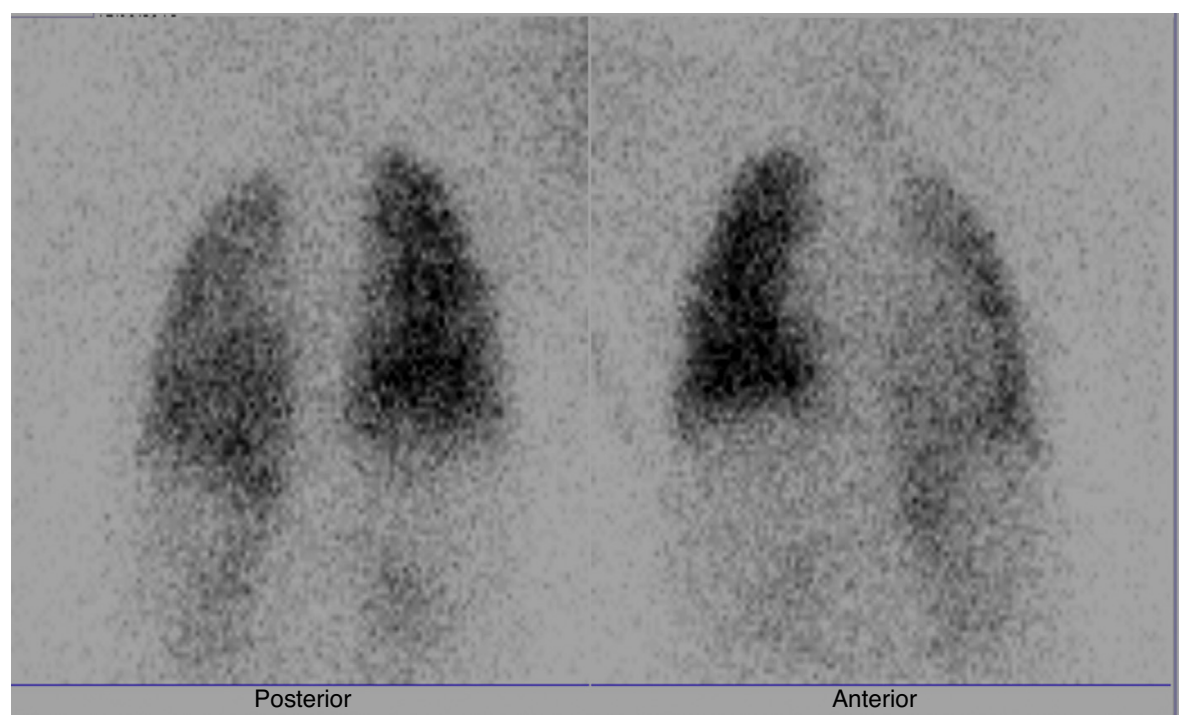

Figura 2 Gammagrafía pulmonar de perfusión con ${ }^{99 m} \mathrm{Tc}-\mathrm{MAA}$ en un paciente con disminución en la perfusión izquierda, posterior al implante del Amplatzer Duct Occluder. 
Tabla 2 Relación entre la perfusión pulmonar y la presencia o ausencia de protrusión del dispositivo

\begin{tabular}{llll}
\hline Perfusión & $\begin{array}{l}\text { Con protrusión } \\
\text { del dispositivo } \\
(\mathrm{n}=6)\end{array}$ & $\begin{array}{l}\text { Sin protrusión } \\
\text { del dispositivo } \\
(\mathrm{n}=24)\end{array}$ & $\begin{array}{l}\text { Valor de } \\
\mathrm{p}\end{array}$ \\
\hline $\begin{array}{l}\text { Pulmón izquierdo } \\
\text { Mediana }\end{array}$ & 42.77 & 45.25 & \\
Mínimo & 39.97 & 37.76 & 0.27 \\
Máximo $\quad 47$ & 61 & \\
Pulmón derecho & & \\
Mediana & 57.24 & 54.77 & 0.29 \\
Mínimo & 53.23 & 38.64 & \\
Máximo & 60 & 62 & \\
\hline
\end{tabular}

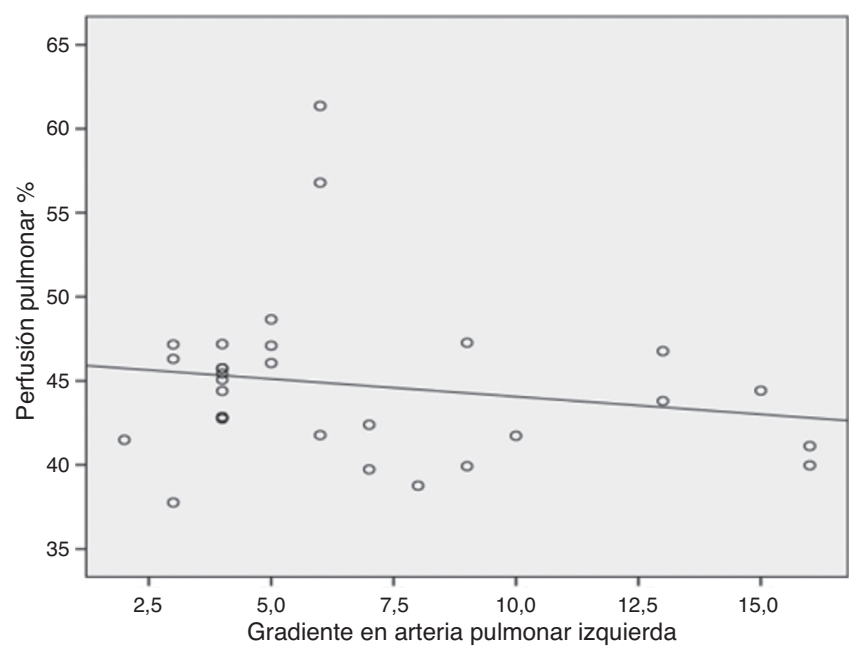

Figura 3 Correlación de la perfusión pulmonar izquierda y el gradiente en la arteria pulmonar izquierda.

resultó estadísticamente significativa $(p=0.28)$. El tamaño del ADO y un incremento $>2 \mathrm{~mm}$ al diámetro mínimo del CAP no fueron estadísticamente significativos $(p=0.22$ y 0.35 , respectivamente) en aquellos pacientes con disminución en la perfusión pulmonar izquierda. En 3 de los 5 pacientes con conducto arterioso tipo tubular, se observó reducción del flujo pulmonar izquierdo.

\section{Discusión}

Desde la primera descripción del cierre percutáneo del conducto arterioso, 3 décadas de experiencia y seguimiento han establecido la seguridad y eficacia de este procedimiento como una alternativa al cierre quirúrgico ${ }^{18-20}$. Habitualmente, los CAP pequeños $(<2 \mathrm{~mm})$ son ocluidos con coils de Gianturco o coils de liberación controlada ${ }^{1-3}$. El $A D O$ es empleado con éxito en el cierre de virtualmente todos los tipos morfológicos del CAP y ofrece muchas ventajas en comparación con otros oclusores ${ }^{4-6,10}$. Sin embargo, en niños pequeños se han reportado la embolización del oclusor, la obstrucción de la luz aórtica o de la rama pulmonar izquierda y otras complicaciones ${ }^{3,7-10}$. Los efectos adversos asociados con el ADO son ligeramente mayores en niños con peso $<10 \mathrm{~kg}^{4,6,10}$. Varios estudios muestran que los gradientes en la API no difieren tras la oclusión del CAP con el ADO, al compararlos con los gradientes preoclusión $n^{1,3-5,16}$.

Algunos estudios analizaron la correlación entre la imágenes ecocardiográficas de protrusión del dispositivo hacia estructuras vasculares adyacentes y el gradiente medido por Doppler y la distribución del flujo sanguíneo pulmonar posterior al cierre percutáneo del $\mathrm{CAP}^{11-13}$, concluyendo que no hay correlación entre las imágenes ecocardiográficas bidimensionales de protrusión del dispositivo y el gradiente de presión por velocidad de flujo Doppler través de la $\mathrm{API}^{16}$. Soares et al. mencionan que el índice de velocidad Doppler es altamente predictivo de asimetría en la perfusión pulmonar $^{13}$.

Estudios sistemáticos que incluyen la gammagrafía pulmonar de perfusión en la evaluación rutinaria de pacientes sometidos a cierre percutáneo del CAP con diferentes dispositivos encuentran un riesgo potencial de alteraciones en la perfusión pulmonar izquierda ${ }^{11-14,21,22}$. Estudios en pacientes sin anomalías cardiacas muestran una distribución normal del flujo sanguíneo pulmonar del $52.5 \%$ y del $47.5 \%( \pm 2.1 \%)$ para el pulmón derecho e izquierdo ${ }^{23}$, considerando un valor de corte $>45 \%$ para una perfusión pulmonar izquierda normal. No existen suficientes datos de una distribución normal del flujo sanguíneo pulmonar en las diferentes edades pediátricas ${ }^{24}$. En este estudio consideramos una disminución del $10 \%$ en la perfusión pulmonar izquierda como anormal (perfusión pulmonar izquierda $<40 \%$ del flujo sanguíneo total). La alta incidencia de alteraciones en la perfusión pulmonar posterior al cierre del CAP referida por diversos autores podría deberse a la falta de una definición adecuada de perfusión pulmonar anormal o por tratarse de serie de casos no consecutivos ${ }^{21,22,25}$.

En este estudio detectamos una disminución en la perfusión pulmonar izquierda mediante la gammagrafía pulmonar de perfusión en 5/30 (16.6\%) pacientes, utilizando un valor de corte $<40 \%$ según lo sugerido por otros estudios $^{14,15,22}$. La media de edad, el peso y la longitud del CAP fueron estadísticamente significativos en los pacientes con perfusión pulmonar disminuida ${ }^{21,25}$, y el porcentaje de pacientes con disminución en la perfusión pulmonar izquierda no se correlacionó con la $V_{\text {máx }}$ en la API, medida por ecocardiografía Doppler.

Las alteraciones en la perfusión pulmonar utilizando el ADO varían en una proporción del 17-33\% ${ }^{14,21,22}$. Polat et al. consideran que una longitud del conducto $<5.8 \mathrm{~mm}$ y una relación diámetro ampolla ductal/longitud ampolla ductal $\geq 1.9$ son los mejores valores de corte obtenidos por curvas ROC, para discriminar una perfusión pulmonar disminuida ${ }^{21}$. Aunque la edad y el peso de los pacientes y la longitud del CAP pueden ser factores de riesgo para una inadecuada perfusión pulmonar izquierda, se considera que el determinante primario podría ser la anatomía ductal ${ }^{14,15,22}$. Se identifican factores de riesgo asociados con gammagrafías anormales, y se confirma la mejoría espontánea de la perfusión pulmonar izquierda en una proporción significativa de pacientes durante su seguimiento $(65.7 \%)^{25,26}$.

\section{Conclusiones}

Este estudio demuestra que el cierre del CAP con el ADO está asociado con un riesgo potencial de alteraciones en 
la perfusión pulmonar izquierda en 5/30 (16.6\%) de los pacientes, utilizando la gammagrafía pulmonar de perfusión. Esto ocurre más a menudo en niños pequeños y con bajo peso, y está muy relacionado con la anatomía ductal. Conductos cortos y tubulares son más propensos a desarrollar esta complicación. No existe una estrecha correlación entre la gammagrafía pulmonar y la ecocardiografía Doppler para establecer alteraciones en la perfusión pulmonar.

\section{Limitaciones}

Es importante mencionar las limitaciones de este estudio, incluyendo su naturaleza retrospectiva y un tamaño de muestra pequeño. Es también importante darse cuenta de que esto no prueba una relación causa-efecto entre una perfusión izquierda anormal y el cierre del CAP con el dispositivo, considerando la ausencia de estudios de perfusión preintervención y la falta de correlación con otros modos de valoración. El significado clínico de nuestros datos requiere de una validación con nuevos estudios prospectivos y un seguimiento a largo plazo.

\section{Responsabilidades éticas}

Protección de personas y animales. Los autores declaran que los procedimientos seguidos se conformaron a las normas éticas del comité de experimentación humana responsable y de acuerdo con la Asociación Médica Mundial y la Declaración de Helsinki.

Confidencialidad de los datos. Los autores declaran que han seguido los protocolos de su centro de trabajo sobre la publicación de datos de pacientes.

Derecho a la privacidad y consentimiento informado. Los autores han obtenido el consentimiento informado de los pacientes y/o sujetos referidos en el artículo. Este documento obra en poder del autor de correspondencia.

\section{Financiación}

No se recibió patrocinio de ningún tipo para llevar a cabo este estudio.

\section{Conflicto de intereses}

Los autores declaran no tener conflicto de intereses.

\section{Bibliografía}

1. Pass RH, Hijazi ZM, Hsu DT, et al. Multicenter USA Amplatzer patent ductus arteriosus occlusion device trial: Initial and oneyear results. J Am Coll Cardiol. 2004;44:513-9.

2. Faella HJ, Hijazi ZM. Closure of the patent ductus arteriosus with de amplatzer PDA device: Inmediate results of the international clinical trial. Catheter Cardiovasc Interv. 2000;51: 50-4.

3. Masura J, Walsh KP, Thanopoulos B, et al. Catheter closure of moderate of moderate- to large-sized patent ductus arteriosus using the new Amplatzer duct occluder: Immediate and short term results. J Am Coll Cardiol. 1998;31:878-82.

4. Bilkis AA, Alwi M, Hasri S, et al. The Amplatzer duct occluder: Experience en 209 patient. J Am Coll Cardiol. 2001;37: 258-61.

5. Masura J, Tittel P, Gavora P, et al. Long-term outcome of transcatheter patent ductus arteriosus closure using Amplatzer duct occluders. Am Heart J. 2006;151, 755.e7-755.e10.

6. Al-Ata J, Arfi AM, Hussain A, et al. The efficacy and safety of the Amplatzer ductal occluder in young children and infants. Cardiol Young. 2005;15:279-85.

7. Fischer G, Stich J, Grabitz R, et al. Transcatheter closure of persistent ductus arteriosus in infants using the Amplatzer duct occluder. Heart. 2001;86:444-7.

8. Parra R, Cruz A, Toxqui A, et al. Cierre percutáneo del conducto arterioso persistente con dispositivo Amplatzer en niños: resultados inmediatos y a un año. Arch Cardiol Mex. 2009;79: 114-20.

9. Butera G, de Rosa G, Chessa M, et al. Transcatheter closure of persistent ductus arteriosus with the Amplatzer duct occluder in very young symptomatic children. Heart. 2004;90: 1467-70.

10. Parra R, Cruz A, Rebolledo V, et al. Cierre transcatéter del conducto arterioso persistente con dispositivo de Amplatzer en niños menores de un año. Rev Esp Cardiol. 2009;62: 867-74.

11. Dessy H, Hermus JPS, van den Heuvel F, et al. Echocardiographic and radionuclide pulmonary blood flow patterns after transcatheter closure of patent ductus arteriosus. Circulation. 1996;94:126-9.

12. Sreeram N, Tofeig $M$, Walsh $K P$, et al. Lung perfusion studies after detachable coil occlusion of persistent arterial duct. Heart. 1999;82:642-5.

13. Soares AM, Aiello VD, Andrade JL, et al. Doppler flow evaluation can anticipate abnormal left lung perfusion after transcatheter closure of patent ductus arteriosus. Eur Heart J. 2004;25:1927-33.

14. Kharouf R, Heitschmidt M, Hijazi ZM. Pulmonary perfusion scans following transcatheter patent ductus arteriosus closure using Amplatzer devices. Catheter Cardiovasc Interv. 2011;77:664-70.

15. Polat TB, Celebi A, Hacimahmutoglu S, et al. Radionuclide evaluation of lung perfusion after transcatheter closure of patent ductus arteriosus: analysis of frequently used two different types of coils. Anadolu Kardiyol Derg. 2011;11:218-24.

16. Gowda S, Kutty S, Ebeid M, et al. Preclosure pressure gradients predict patent ductus arteriosus patients at risk for later pulmonary artery stenosis. Pediatr Cardiol. 2009;30:883-7.

17. Krichenko A, Benson LN, Burrows P, et al. Angiographic classification of the isolated, persistently ductus arteriosus and implications for percutaneous catheter occlusion. Am J Cardiol. 1989;63:877-80.

18. Zabal C. Usefulness of Amplatzer devices. Arch Cardiol Mex. 2009;79:81-2.

19. San Luis-Miranda R, Arias-Monroy LG, Peralta-Pedrero ML, et al. Clinical guide practice. Patent ductus arteriosus. Rev Med Inst Mex Seguro Soc. 2012;50:450-63.

20. Cervantes-Salazar J, Calderón-Colmenero J, Ramírez-Marroquín $\mathrm{S}$, et al. Pediatric cardiovascular surgical data base registry in México. First report. Rev Invest Clin. 2013;65:476-82.

21. Polat TB, Celebi A, Hacimahmutoglu $S$, et al. Lung perfusion studies after transcatheter closure persistent ductus arteriosus with the Amplatzer duct occluder. Catether Cardiovasc Interv. 2010;76:418-24.

22. Evangelista JK, Hijazi ZM, Geggel RL, et al. Effect of multiple coil closure of patent ductus arteriosus on blood flow to the left lung as determined by lung perfusion scans. Am J Cardiol. 1997;80:242-4. 
23. Treves ST, Packarad AB. Lungs. En: Treves ST, editor. Pediatric nuclear medicine/PET. New York: Verlag; 2007. p. 87-127.

24. Johnson K. Ventilation and perfusion scanning in children. Paediatric Respir Rev. 2000;1:347-53.

25. Kramoh EK, Miró J, Brigas JL, et al. Differential pulmonary perfusion scan after percutaneous occlusion of the patent ductus arteriosus: One-decade consecutive longitudinal study from a single institution. Pediatr Cardiol. 2008;29: 918-22.

26. Demir F, Celebi A, Saritas T, et al. Long-term follow-up results of lung perfusion studies after transcatheter closure of patent ductus arteriosus. Cong Heart Dis. 2013;8:159-66. 